\title{
Pressure overload-induced cardiac remodeling and dysfunction in the absence of interleukin 6 in mice
}

\author{
N Chin Lai, Mei Hua Gao, Eric Tang, Ruoying Tang, Tracy Guo, Nancy D Dalton, Aihua Deng and Tong Tang
}

Congestive heart failure is associated with increased expression of pro-inflammatory cytokines, and the levels of these cytokines correlate with heart failure severity and prognosis. Chronic interleukin 6 (IL-6) stimulation leads to left ventricular (LV) hypertrophy and dysfunction, and deletion of IL- 6 reduces LV hypertrophy after angiotensin II infusion. In this study, we tested the hypothesis that IL- 6 deletion has favorable effects on pressure-overloaded hearts. We performed transverse aortic constriction on IL-6-deleted (IL6KO) mice and C57BL/6J mice (CON) to induce pressure overload. Pressure overload was associated with similar LV hypertrophy, dilation, and dysfunction in CON and IL6KO mice. Re-activation of the fetal gene program was also similar in pressure-overloaded CON and IL6KO mice. There were no differences between CON and IL6KO mice in LV fibrosis or expression of extracellular matrix proteins after pressure overload. In addition, no group differences in apoptosis or autophagy were seen. These data indicate that IL- 6 deletion does not block LV remodeling and dysfunction induced by pressure overload. Attenuated content of IL-11 appears to be a compensatory mechanism for IL-6 deletion in pressure-overloaded hearts. We infer from these data that limiting availability of IL-6 alone is not sufficient to attenuate LV remodeling and dysfunction in failing hearts.

Laboratory Investigation (2012) 92, 1518-1526; doi:10.1038/labinvest.2012.97; published online 23 July 2012

KEYWORDS: fibrosis; heart failure; hypertrophy; IL-6; inflammatory cytokine; LV function; pressure overload

Congestive heart failure is an inexorable disease associated with high morbidity and mortality, ${ }^{1}$ despite advanced pharmaceutical and device therapies. Chronic pressure overload, as seen in persistent hypertension, is a major risk factor for heart failure. ${ }^{2}$ Although the underlying mechanisms are not fully understood, pro-inflammatory cytokines may be of mechanistic importance for left ventricular (LV) dysfunction and dilation. ${ }^{3}$

Serum levels of the pro-inflammatory interleukin 6 (IL-6) family cytokines (IL-6, leukemia inhibitory factor (LIF), ciliary neurotrophic factor (CNTF), oncostatin $M$ and cardiotrophin 1) are elevated in patients with heart failure regardless of etiology. ${ }^{4-10}$ Clinical and preclinical studies also show that increased levels of these pro-inflammatory IL-6 family cytokines correlate with high risk of developing LV hypertrophy, dilation, dysfunction and fibrosis. ${ }^{4-6,11-14}$ In addition, IL-6 perfusion directly inhibits contractility in isolated papillary muscle. ${ }^{15}$ Impaired contractility likely results from decreased SERCA2a expression after IL-6 stimulation. ${ }^{16}$ In vivo studies show that chronic IL-6 infusion is associated with LV hypertrophy, fibrosis and dysfunction. ${ }^{17,18}$
These observations indicate that increased IL-6 levels impair LV function. Finally, deletion of IL-6 reduces LV hypertrophy after angiotensin II infusion. ${ }^{19}$ Our hypothesis is that reduced IL-6 levels will decrease LV hypertrophy and improve function of the pressure-overloaded hearts. We induced pressure overload by transverse aortic constriction (TAC) in a murine line with IL-6 deletion to test this hypothesis.

\section{MATERIALS AND METHODS \\ Animals}

This study was approved by Institutional Animal Care and Use Committee at the VA San Diego Healthcare System, in accordance with NIH and AAALAC guidelines. Male and female mice with germline IL-6 deletion (IL6KO; in congenic C57BL/6J background) were purchased from the Jackson Laboratories (Bar Harbor, ME, USA). Two-month-old IL6KO mice were used for the study. Age- and sex-matched wildtype C57BL/6J mice were used as control (CON). Deletion of IL-6 gene was confirmed by PCR using mouse tail genomic DNA as template (Supplementary Figure S1A). Absence of IL-6 mRNA expression and protein expression were further

Department of Medicine, University of California San Diego, VA San Diego Healthcare System, San Diego, CA, USA

Correspondence: Dr T Tang, VA San Diego Healthcare System (111A), 3350 La Jolla Village Drive, San Diego, CA 92161, USA.

E-mail: ttang@vapop.ucsd.edu

Received 25 November 2011; revised 15 April 2012; accepted 24 April 2012 
confirmed by quantitative real-time RT-PCR and enzymelinked immunosorbent assay (ELISA), respectively (Supplementary Figure S1).

\section{Pressure Overload}

Pressure overload was induced by TAC as previously reported..$^{20}$ Briefly, anesthetized mice were intubated and ventilated (pressure-controlled) with anesthesia maintained with $1 \%$ isoflurane in oxygen. The second intercostal space at the left upper sternal border was blunt dissected to expose the aortic arch. A 7-0 silk tie was applied against a 27-gauge needle between the innominate and left carotid artery. The needle was then removed, which leads to $\sim 90 \%$ lumen narrowing. Surgery was performed without knowledge of group identity.

\section{Echocardiography}

Mouse anesthesia was induced with $5 \%$ isoflurane (at a flow rate of $11 /$ min oxygen) and maintained with $1 \%$ isoflurane in oxygen. Echocardiographic images were obtained using a $16 \mathrm{~L} \mathrm{MHz} \mathrm{linear} \mathrm{probe} \mathrm{and} \mathrm{Sonos} 5500^{\circledR}$ Echocardiograph system (Philips Healthcare, Andover, MA, USA). LV chamber dimensions and LV fractional shortening were acquired and analyzed without knowledge of group identity.

\section{In Vivo Hemodynamics}

A $1.4 \mathrm{~F}$ conductance-micromanometer catheter (Millar Instruments) was inserted into the right carotid artery to access the LV cavity on anesthetized mice $(80 \mathrm{mg} / \mathrm{kg}$ sodium pentobarbital, i.p.). $\mathrm{LV}$ pressure development $(\mathrm{LV}+\mathrm{dP} / \mathrm{dt}$ ), relaxation $(-\mathrm{dP} / \mathrm{dt})$ and end-systolic pressure-volume relationship (ESPVR) were determined as previously described. $^{20}$

\section{Necropsy}

Body and LV weight (including the septum), and tibial length were measured at necropsy.

A short axis midwall LV ring was fixed in formalin; the remaining portion of $\mathrm{LV}$ was quickly frozen in liquid nitrogen and stored at $-80^{\circ} \mathrm{C}$.

\section{Histology}

Formalin-fixed LV samples were dehydrated and embedded in paraffin. Dewaxed LV sections $(5 \mu \mathrm{m})$ were rehydrated and stained with hematoxylin and eosin. To measure collagen area, rehydrated LV sections were stained with picrosirius red. Fractional collagen area was calculated as percentage of picrosirius red-stained collagen area using NIH ImageJ software.

\section{Quantitative RT-PCR}

Total RNA was extracted, purified and treated with RNasefree DNase to eliminate potential genomic DNA contamination.
Quantitative real-time RT-PCR was conducted as previously described. $^{20}$

\section{Western Blotting}

LV homogenates were used for western blotting as previously described, ${ }^{20}$ using the following antibodies: Bcl2 (Cat \#sc-7382, Santa Cruz Biotechnology, Santa Cruz, CA, USA), $\alpha$-smooth muscle actin (Cat \#A5228, Sigma, St Louis, MO, USA), periostin (Cat \#AF2955, R\&D Systems, Minneapolis, MN, USA), cathepsin D (Cat \#AF1029, R\&D Systems), LC3 (Cat \#2775, Cell Signaling Technology, Danvers, MA, USA), phospho-STAT3 (Cat \#9145, Cell Signaling Technology), and STAT3 (Cat \#9139, Cell Signaling Technology). Equal loading of samples and even transfer efficiency were monitored by re-probing stripped blots with the antibody to glyceraldehyde 3-phosphate dehydrogenase (Cat \#10R-G109A, Fitzgerald Industries International, Acton, MA, USA). Quantification of protein expression was performed using Gel-Pro ${ }^{\circledR}$ Analyzer (Media Cybernetics, Silver Spring, MD, USA).

\section{Enzyme-Linked Immunosorbent Assay}

Serum IL-6 and IL-11 levels in CON and IL6KO mice were measured using mouse IL-6 ELISA Ready-SET-Go kit (Cat \#88-7064-22, eBioscience, San Diego, CA, USA) and mouse IL-11 ELISA kit (Cat \#ELM-IL11-001, RayBiotech, Norcross, GA, USA).

\section{Terminal dUTP Nick End-labeling (TUNEL) Assay}

Apoptotic cells were identified by TUNEL assay using CardioTACS In situ Apoptosis Detection Kit (R\&D Systems) as described previously. ${ }^{21}$

\section{Statistical Analysis}

Results are shown as mean \pm s.e. Data were analyzed using two-way ANOVA followed by Bonferroni post hoc test (for comparison of CON $v$ s IL6KO mice, 2 weeks after TAC). The null hypothesis was rejected when $P<0.05$.

\section{RESULTS \\ LV Function after Pressure Overload}

Echocardiography showed reduced LV fractional shortening in CON mice 2 weeks after pressure overload (Table 1, Supplementary Figure S2). These mice also had increased LV end-diastolic diameter and wall thickness, which was associated with increased mRNA expression of IL-6 in LV samples (no TAC: $100 \pm 10 \%, n=8$; 2 weeks after TAC: $695 \pm 160 \%$, $n=8 ; P<0.002)$. IL6KO mice showed a similar degree reduction of LV fractional shortening 2 weeks after pressure overload (Table 1). There were no group differences in LV chamber dimensions between CON and IL6KO mice either before or 2 weeks after pressure overload. In addition, echocardiography showed similar changes in LV chamber dimensions and LV fractional shortening in CON and IL6KO mice after 4 weeks pressure overload (Table 2). In vivo hemodynamics study was performed to assess LV function 4 weeks 
Table 1 Echocardiography measurements 2 weeks after TAC

\begin{tabular}{|c|c|c|c|c|c|c|c|}
\hline & \multicolumn{2}{|c|}{ No TAC } & \multicolumn{2}{|c|}{2 Weeks after TAC } & \multicolumn{3}{|c|}{$P$-value } \\
\hline & $\operatorname{CON}(n=10)$ & IL6KO $(n=10)$ & $\operatorname{CON}(n=9)$ & IL6KO $(n=6)$ & Interaction & IL-6 deletion effect & TAC effect \\
\hline HR (b.p.m.) & $627 \pm 10$ & $622 \pm 15$ & $631 \pm 14$ & $606 \pm 18$ & NS & NS & NS \\
\hline LVEDD $(\mathrm{mm})$ & $3.2 \pm 0.1$ & $3.1 \pm 0.1$ & $3.7 \pm 0.2$ & $3.6 \pm 0.1$ & NS & NS & $<0.002$ \\
\hline $\operatorname{LVESD}(\mathrm{mm})$ & $1.8 \pm 0.1$ & $1.8 \pm 0.1$ & $2.6 \pm 0.3$ & $2.5 \pm 0.2$ & NS & NS & $<0.0005$ \\
\hline IVSd (mm) & $0.6 \pm 0.01$ & $0.6 \pm 0.01$ & $0.8 \pm 0.03$ & $0.9 \pm 0.04$ & NS & NS & $<0.0001$ \\
\hline FS (\%) & $44 \pm 1$ & $44 \pm 1$ & $32 \pm 4$ & $31 \pm 4$ & NS & NS & $<0.0001$ \\
\hline
\end{tabular}

Abbreviations: FS, fractional shortening; HR, heart rate; IVSd, interventricular septum thickness in diastole; LVEDD, left ventricular end-diastolic diameter; LVESD, left ventricular end-systolic diameter; NS, not significant; PWTd, posterior wall thickness in diastole; TAC, transverse aortic constriction.

Values shown are mean \pm s.e.m. Probability values are from two-way ANOVA for interaction effect, IL- 6 deletion effect and TAC effect.

Table 2 Echocardiography measurements 4 weeks after TAC

\begin{tabular}{|c|c|c|c|c|c|c|c|}
\hline & $\operatorname{CON}(n=8)$ & IL6KO $(n=8)$ & $\operatorname{CON}(n=5)$ & IL6KO $(n=5)$ & Inter & IL-6 deletion effect & TAC effect \\
\hline HR (b.p.m.) & $528 \pm 25$ & $521 \pm 28$ & $463 \pm 17$ & $474 \pm 31$ & NS & NS & NS \\
\hline LVEDD (mm) & $3.5 \pm 0.1$ & $3.3 \pm 0.1$ & $3.9 \pm 0.2$ & $3.6 \pm 0.1$ & NS & NS & $<0.01$ \\
\hline IVSd (mm) & $0.6 \pm 0.01$ & $0.6 \pm 0.01$ & $0.8 \pm 0.03$ & $0.9 \pm 0.05$ & NS & NS & $<0.0001$ \\
\hline FS (\%) & $42 \pm 3$ & $41 \pm 2$ & $25 \pm 3$ & $29 \pm 4$ & NS & NS & $<0.0001$ \\
\hline
\end{tabular}

Abbreviations: FS, fractional shortening; HR, heart rate; IVSd, interventricular septum thickness in diastole; LVEDD, left ventricular end-diastolic diameter; LVESD, left ventricular end-systolic diameter; NS, not significant; PWTd, posterior wall thickness in diastole; TAC, transverse aortic constriction.

Values shown are mean \pm s.e.m. Probability values are from two-way ANOVA for interaction effect, IL- 6 deletion effect and TAC effect.

Table 3 Morphometric measurements

\begin{tabular}{|c|c|c|c|c|c|c|c|}
\hline & \multicolumn{2}{|c|}{ No TAC } & \multicolumn{2}{|c|}{2 Weeks after TAC } & \multicolumn{3}{|c|}{$P$-value } \\
\hline & $\operatorname{CON}(n=5)$ & IL6KO $(n=5)$ & $\operatorname{CON}(n=9)$ & IL6KO $(n=6)$ & Interaction & IL-6 deletion effect & TAC effect \\
\hline Body weight (g) & $22.5 \pm 0.3$ & $23.6 \pm 1.1$ & $25.5 \pm 0.7$ & $24.5 \pm 0.5$ & NS & NS & $<0.02$ \\
\hline LV weight (mg) & $87 \pm 3$ & $88 \pm 4$ & $154 \pm 16$ & $157 \pm 12$ & NS & NS & $<0.0001$ \\
\hline Kidney (mg) & $322 \pm 10$ & $331 \pm 18$ & $300 \pm 14$ & $289 \pm 7$ & NS & NS & $<0.04$ \\
\hline Spleen (mg) & $64 \pm 7$ & $85 \pm 9$ & $80 \pm 3$ & $70 \pm 4$ & NS & NS & NS \\
\hline Tibial length (mm) & $17.2 \pm 0.1$ & $17.4 \pm 0.2$ & $17.0 \pm 0.3$ & $16.8 \pm 0.1$ & NS & NS & NS \\
\hline LV/BW (mg/g) & $3.9 \pm 0.1$ & $3.8 \pm 0.3$ & $6.1 \pm 0.7$ & $6.4 \pm 0.5$ & NS & NS & $<0.001$ \\
\hline
\end{tabular}

Abbreviations: BW, body weight; NS, not significant; TAC, transverse aortic constriction; TL, tibial length.

Values shown are mean \pm s.e.m. Probability values are from two-way ANOVA for interaction effect, IL- 6 deletion effect and TAC effect. 
after pressure overload in detail. There were no group differences in $\mathrm{LV}+\mathrm{dP} / \mathrm{dt}(\mathrm{CON}: 4159 \pm 557 \mathrm{mmHg} / \mathrm{s}$, $n=10$; IL6KO: $3521 \pm 396 \mathrm{mmHg} / \mathrm{s}, n=8 ; P=0.4)$ and LV $-\mathrm{dP} / \mathrm{dt} \quad(\mathrm{CON}: \quad-4901 \pm 891 \mathrm{mmHg} / \mathrm{s}, \quad n=10 ; \quad$ IL6KO: $-3935 \pm 787 \mathrm{mmHg} / \mathrm{s}, n=8 ; P=0.4) 4$ weeks after pressure overload. ESPVR, a measure relatively less dependent on load conditions, was not altered either (CON: $3.4 \pm 0.7 \mathrm{mmHg} / \mu \mathrm{l}$, $n=9$; IL6KO: $2.3 \pm 0.5 \mathrm{mmHg} / \mu \mathrm{l}, \quad n=9 ; \quad P=0.3)$. Taken together, these data indicate that IL-6 deletion does not attenuate LV dilation and dysfunction associated with pressure overload.

\section{Size}

Pressure overload was associated with increased LV weight, LV weight/body weight ratio, LV weight/tibial length ratio in CON mice (Table 3; Figures 1a and $\mathrm{b}$ ). The increase in lung weight after TAC indicates severe pulmonary congestion. IL6KO mice showed similar increases as CON mice in LV weight, lung weight, LV weight/body weight ratio and LV weight/tibial length ratio after pressure overload (Table 3). By quantitative RT-PCR, we found comparable mRNA expression of the fetal gene program in LV samples from pressureoverloaded CON and IL6KO mice (Figures 1c-e). There were no differences in cardiac FHL1 mRNA (Figure 1f) or protein content (CON: $762 \pm 97$ densitometric units (du), $n=9$; IL6KO: $658 \pm 120 \mathrm{du}, n=6 ; P=0.51)$. These data indicate that IL-6 deletion does not influence LV hypertrophy induced by pressure overload.

\section{Fibrosis}

As anticipated, pressure overload increased LV fibrosis in CON mice, as shown by increased collagen deposition detected by picrosirius staining (Figure 2a). A similar degree of collagen deposition was found in LV samples from pressure-overloaded IL6KO mice (Figure 2a). Quantification of fractional collagen area showed no difference between CON and IL6KO mice after pressure overload (Figure 2b). There was no group difference in mRNA expression of extracellular matrix proteins collagen $\mathrm{I} \alpha 1$ (Figure $2 \mathrm{c}$ ) and collagen III $\alpha 1$ (Figure 2d). We also found no difference in cardiac protein a

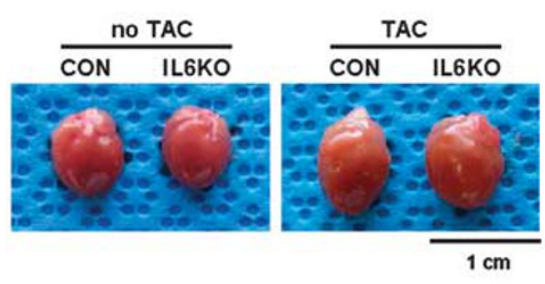

C

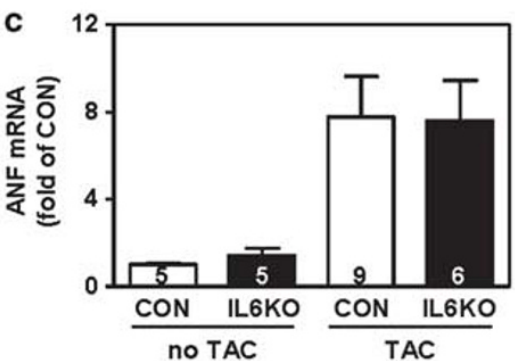

e

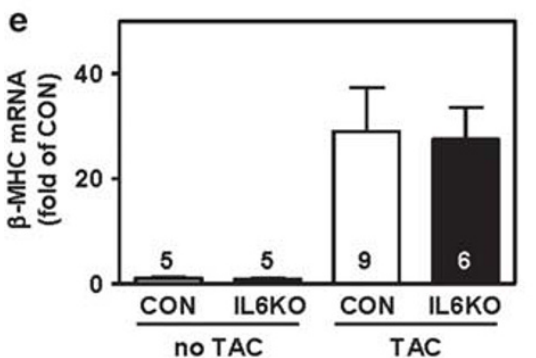

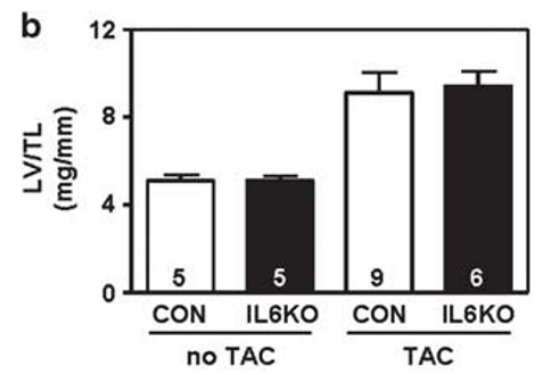
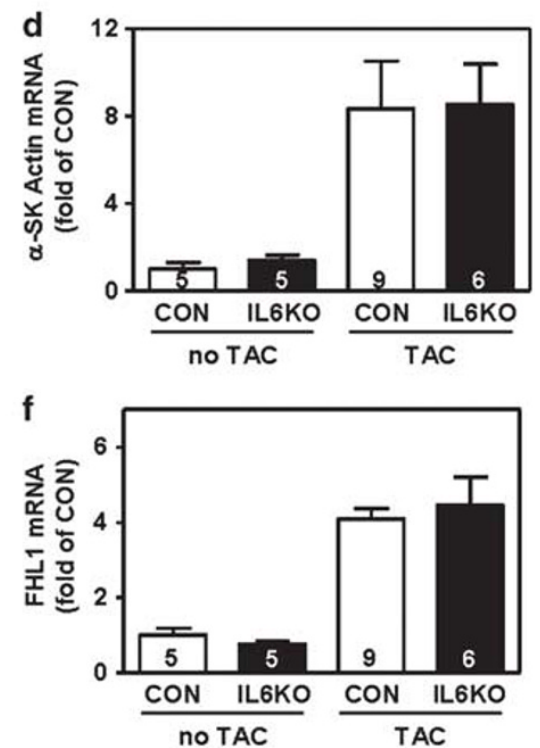

Figure 1 IL-6 deletion was insufficient to block pressure overload-induced LV hypertrophy. (a) Images of hearts from CON and IL6KO mice without TAC and 2 weeks after TAC. (b) There was no group difference in LV/tibial length ratio between CON and IL6KO mice without TAC and 2 weeks after TAC $(P=0.82)$. No group differences in mRNA expression of atrial natriuretic factor (ANF, $P=0.95)(\mathbf{c}), \alpha$-skeletal muscle actin $(\alpha$-SK actin, $P=0.95)(\mathbf{d}), \beta$-myosin heavy chain ( $\beta$-MHC, $P=0.90)$ (e), four-and-a-half LIM domain protein 1 (FHL1, $P=0.61$ ) (f) were observed in LV samples from CON and IL6KO mice 2 weeks after TAC. Probability values are from Bonferroni post hoc test (CON vs IL6KO, 2 weeks after TAC) after two-way ANOVA. Error bars denote 1 s.e.m.; numbers in bars indicate group size. 
a

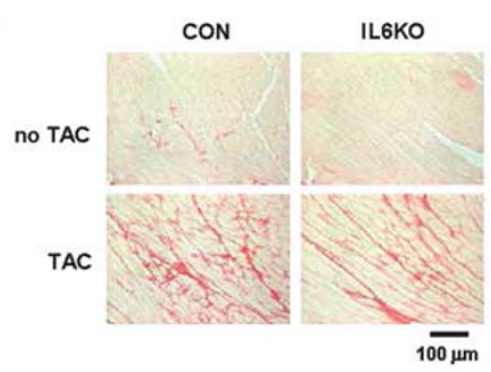

C

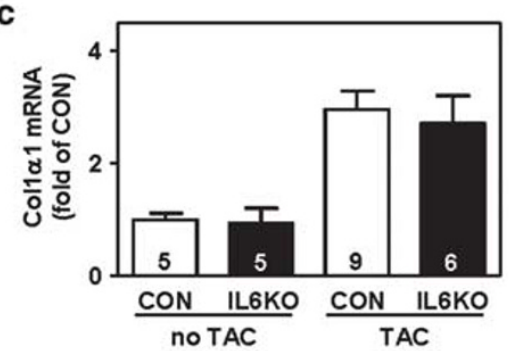

e

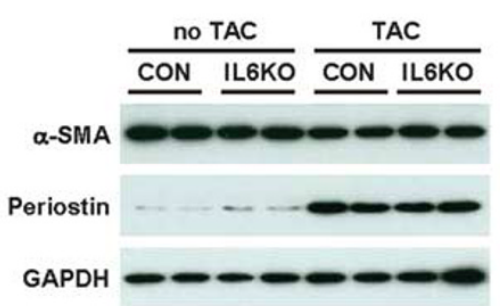

g

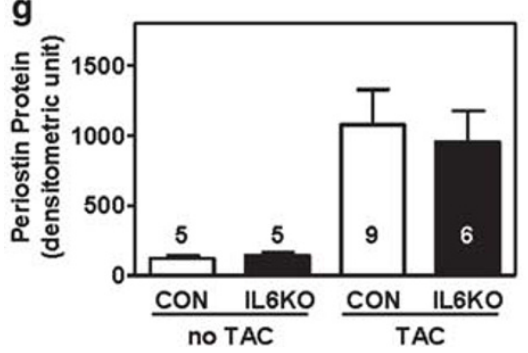

b

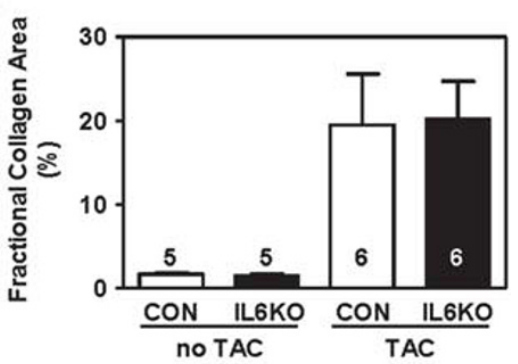

d
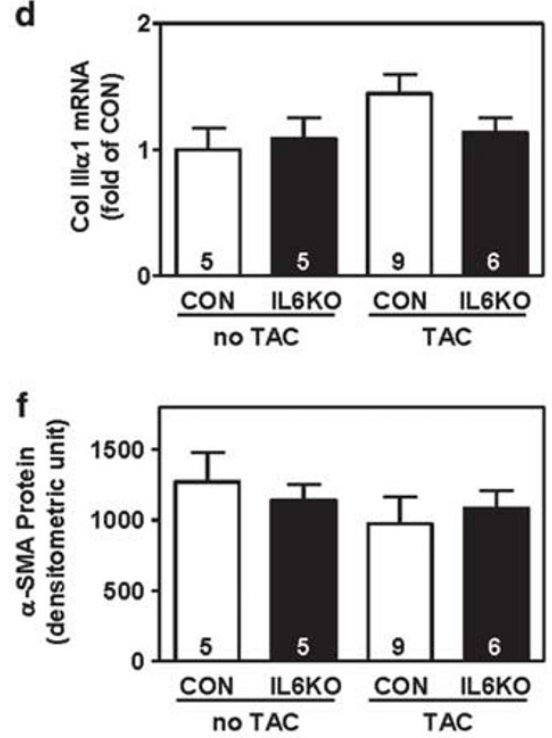

Figure 2 IL-6 deletion was insufficient to block LV fibrosis after pressure overload. (a) Representative photomicrographs show no difference in collagen deposition in LV samples from CON and IL6KO mice without TAC and 2 weeks after TAC. (b) The bar graph summarizes fractional collagen area in LV samples from CON and IL6KO mice without TAC and 2 weeks after TAC $(P=0.92)$. No group differences were observed in mRNA expression of collagen $\mid \alpha 1(P=0.63)(\mathbf{c})$ and collagen IIl $\alpha 1(P=0.13)(\mathbf{d})$ in LV samples from CON and IL6KO mice without TAC and 2 weeks after TAC. (e) Representative western blots showing protein contents of $\alpha$-smooth muscle actin ( $\alpha$-SMA) and periostin in LV samples from CON and IL6KO mice before and 2 weeks after TAC. There was no significant difference in protein expression of $\alpha$-SMA $(P=0.24)(\mathbf{f})$ and periostin $(P=0.74)(\mathbf{g})$. Probability values are from CON vs IL6KO comparison by Bonferroni post hoc test (CON vs IL6KO, 2 weeks after TAC) after two-way ANOVA. Error bars denote 1 s.e.m.; numbers in bars indicate group size.

content of $\alpha$-smooth muscle actin (Figures 2e and $\mathrm{f}$ ) and periostin (Figures $2 \mathrm{e}$ and $\mathrm{g}$ ). These results indicate that IL-6 deletion does not inhibit LV fibrosis induced by pressure overload.

\section{Apoptosis and Autophagy}

Pressure overload increased cardiac myocyte apoptosis (TUNEL staining) (Figure $3 \mathrm{a}$ ), caspase $3 / 7$ activity (Figure $3 \mathrm{~b}$ ) and $\mathrm{Bcl} 2$ protein expression (Figures $3 \mathrm{c}$ and $\mathrm{d}$ ) in $\mathrm{CON}$ mice. IL6KO mice showed similar increases after pressure overload (Figure 3, Supplementary Figure S3). Furthermore, there were no differences in cardiac protein levels of cathepsin D (Figures $3 \mathrm{c}$ and e) and LC3-II (Figures 3c and $\mathrm{f}$ ) between CON and IL6KO mice 2 weeks after TAC. These data suggest that IL-6 deletion does not affect LV apoptosis or autophagy in hearts under pressure overload.

\section{STAT3 Activation}

There were no group differences in mRNA expression of IL-6 receptor (IL-6R) (Figure 4a) and signal transducing 

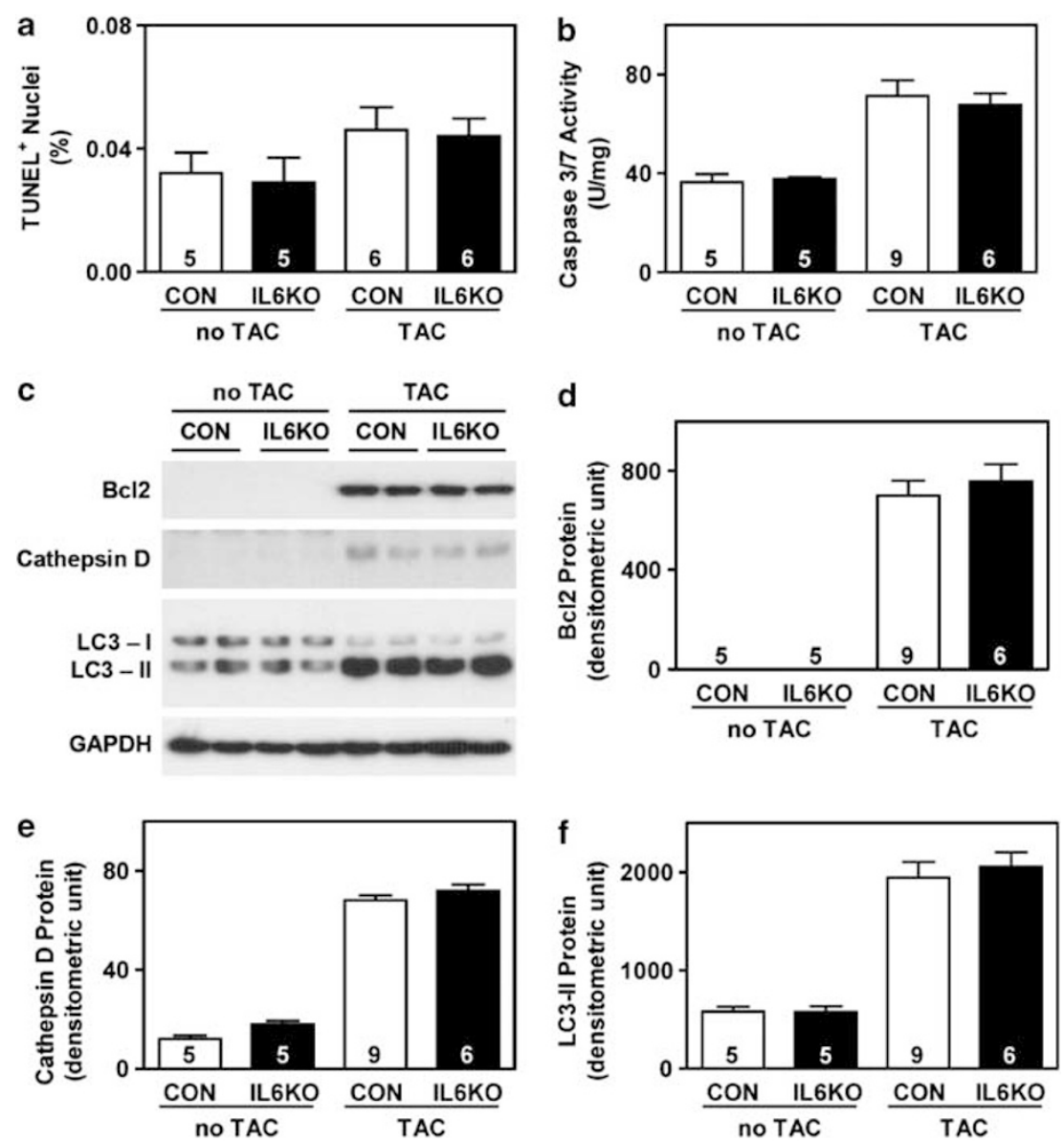

Figure 3 IL-6 deletion was insufficient to suppress apoptosis and had no effect on autophagy in pressure-overloaded hearts. (a) There was no group difference in TUNEL-positive cardiac myocytes in CON and IL6KO mice 2 weeks after TAC $(P=0.83)$. (b) Caspase $3 / 7$ activity was not significantly different in LV samples from CON and IL6KO 2 weeks after TAC $(P=0.90)$. (c) Representative western blots showing protein contents of $B c 12$, cathepsin D and LC3-II in LV samples from CON and IL6KO mice before and 2 weeks after TAC. There was no difference in protein content of Bcl2 $(P=0.55)(\mathbf{d})$, cathepsin $\mathrm{D}(P=0.31)(\mathbf{e})$ and LC3-II $(P=0.64)(\mathbf{f})$ in LV samples from CON and IL6KO mice 2 weeks after pressure overload. Probability values are from CON vs IL6KO comparison by Bonferroni post hoc test (CON vs IL6KO, 2 weeks after TAC) after two-way ANOVA. Error bars denote 1 s.e.m.; numbers in bars indicate group size.

component gp130 (Figure 4b) in LV samples from CON and IL6KO mice either before or 2 weeks after pressure overload. Pressure overload activated STAT3 signaling pathway in CON mice, as shown by increased STAT3 phosphorylation 2 weeks after TAC (Figures $4 \mathrm{c}$ and d). A similar degree of increase in STAT3 phosphorylation was found in LV samples from pressure-overloaded IL6KO mice (Figures $4 \mathrm{c}$ and $\mathrm{d}$ ). No change was found in total STAT3 content (Figures $4 \mathrm{c}$ and e).

\section{Expression of IL-6 Family Members}

Pressure overload was associated with altered cardiac mRNA expression of other IL-6 family members: increased mRNA expression of IL-11 (Figure 4f), LIF (Figure 4g) and CNTF (Supplementary Figure S4A); and decreased mRNA expression of cardiotrophin 1 (Supplementary Figure S4B). Interestingly, IL-6 deletion only attenuated cardiac IL-11 mRNA expression in pressure-overloaded hearts, and had no effects on mRNA expression of LIF (Figure 4g), CNTF (Supplementary Figure S4A) and cardiotrophin 1 (Supplementary
Figure S4B). Decreased serum IL-11 levels were also found in IL6KO mice 2 weeks after TAC (Figure 4h).

\section{DISCUSSION}

Epidemiology and animal studies show that heart failure is associated with increased expression of pro-inflammatory cytokine IL-6., ${ }^{4,22}$ In this study, we showed that cardiac IL-6 expression was increased in pressure-overloaded hearts. However, IL-6 deletion did not influence pressure overloadinduced LV dilation or dysfunction. There were no group differences in LV hypertrophy, fibrosis, apoptosis or autophagy between CON and IL6KO mice after pressure overload. These data indicate that IL-6 deletion is dispensable for LV remodeling and dysfunction in response to pressure overload.

A previous study showed that deletion of IL- 6 attenuated LV hypertrophy induced by infusion of angiotensin II. ${ }^{19}$ However, we found that IL-6 deletion did not affect LV hypertrophy and expression of the fetal gene program in pressure-overloaded hearts (Figure 2). The reason for this 

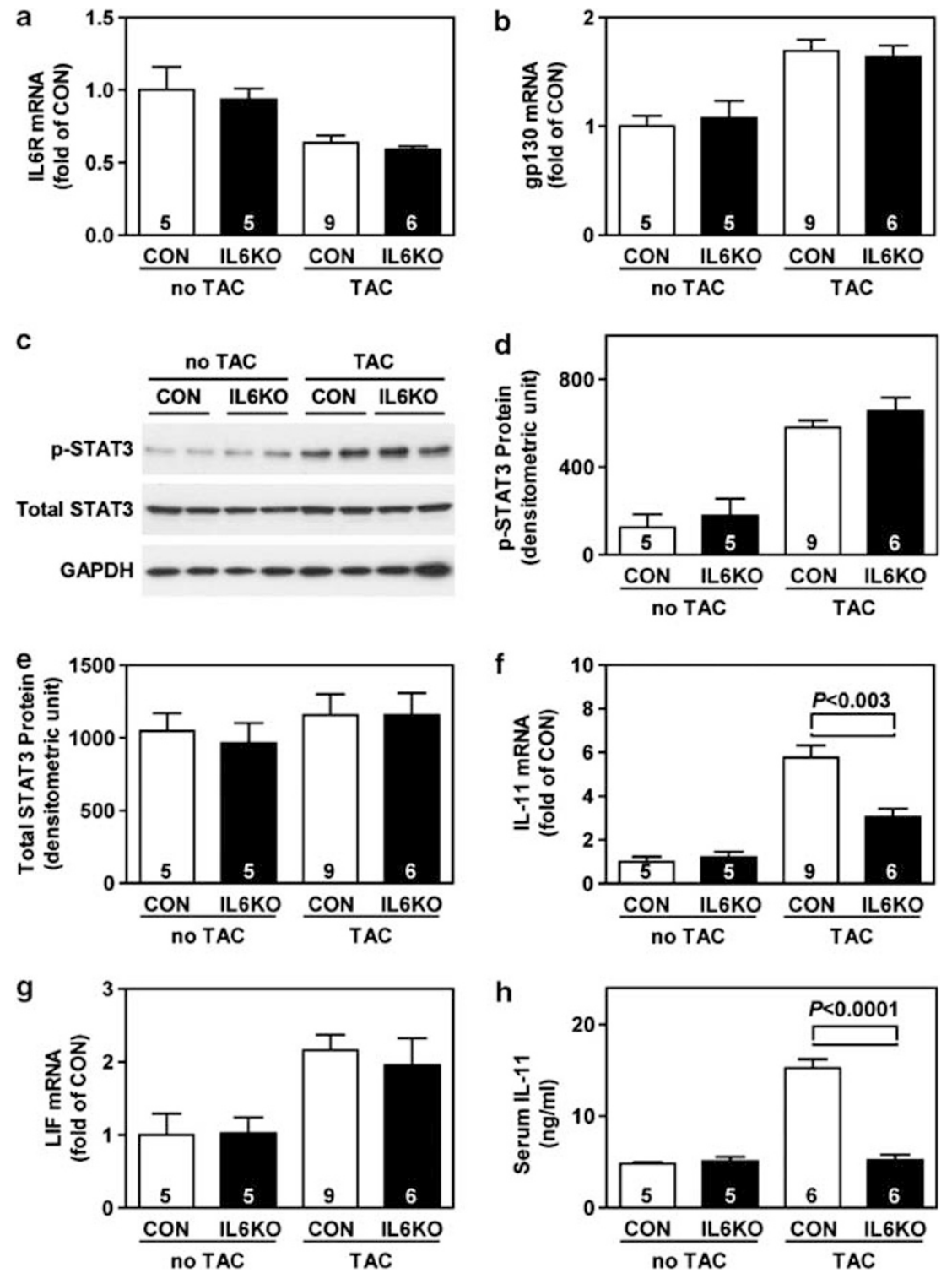

Figure 4 IL-6 deletion was associated with decreased expression of IL-11, which may compensate the IL- 6 deletion effects, in pressure-overloaded hearts. (a) There was no group difference in IL-6 receptor mRNA content in LV samples from CON and IL6KO mice 2 weeks after TAC (P=0.50). (b) There was no group difference in gp130 mRNA content in LV samples from CON and IL6KO mice 2 weeks after TAC $(P=0.76)$. (c) Representative western blots showing protein contents of phospho-STAT3 (p-STAT3) and total STAT3 in LV samples from CON and IL6KO mice before and 2 weeks after TAC. (d) There was no group difference in phospho-STAT3 protein content in LV samples from CON and IL6KO mice 2 weeks after TAC $(P=0.25)$. (e) Total STAT3 protein content was not significantly different in LV samples from CON and IL6KO 2 weeks after TAC $(P=1.00)$. (f) IL-6 deletion was associated with decreased mRNA expression of IL-11 in LV samples from IL6KO mice 2 weeks after TAC. (g) There was no group difference in leukemia inhibitory factor (LIF) mRNA content in LV samples from CON and IL6KO mice 2 weeks after TAC $(P=0.61)$. (h) Decreased serum level of IL-11 was found in IL6KO mice compared with CON mice 2 weeks after TAC. Probability values are from CON vs IL6KO comparison by Bonferroni post hoc test (CON vs IL6KO, 2 weeks after TAC) after two-way ANOVA. Error bars denote 1 s.e.m.; numbers in bars indicate group size.

mismatch is not known, but may be due to differences in models (angiotensin II infusion vs pressure overload). Alternatively, the observed blockade of LV hypertrophy by IL-6 deletion in angiotensin II infusion may reflect the effect of genetic backgrounds, but not the effect of IL-6 deletion. Indeed, control mice in $\mathrm{C} 57 \mathrm{BL} / 6 \mathrm{~N}$ genetic background (Charles River) were used to compared with IL6KO mice (in C57BL/6J genetic background, the Jackson Laboratories) in the previous study. ${ }^{19}$ We have previously shown profound differences in survival and cardiac function change in C57BL/6N and C57BL/6J mice (the Jackson Laboratories) in response to pressure overload. ${ }^{20}$

The IL-6 family cytokines include pro-inflammatory cytokines IL-6, LIF, CNTF, oncostatin M and cardiotropin-1, and anti-inflammatory cytokine IL-11. Previous studies demonstrate that pro-inflammatory IL-6 family members induces 
LV hypertrophy, dilation, dysfunction and fibrosis, ${ }^{4-6,11-14}$ but anti-inflammatory cytokine IL-11 protects cardiac myocytes from ischemia/reperfusion injury ${ }^{23}$ and decreases cardiac fibrosis and apoptosis after myocardial infarction. ${ }^{24}$ Glycoprotein gp130 is a common signal transducing component for receptors for all IL-6 family members, and transduces signals from these cytokines to JAK-STAT3 signaling pathway. The inability of IL- 6 deletion to attenuate LV remodeling and dysfunction after pressure overload may be due to the compensatory effects of other IL- 6 family members. Indeed, deletion of IL- 6 had no effect on cardiac STAT3 phosphorylation either before or after pressure overload (Figures $4 \mathrm{c}$ and $\mathrm{d}$ ). Moreover, IL-6 deletion inhibited pressure overload increased cardiac IL-11 mRNA content and circulating IL-11 level (Figures $4 \mathrm{f}$ and $\mathrm{h}$ ), but did not affect expression of other IL-6 family members. Therefore, attenuated content of IL-11 appears to be a compensatory mechanism for IL-6 deletion in pressure-overloaded hearts.

In addition to pressure overload, myocardial infarction is another major risk factor for congestive heart failure. Although IL-6 reduces apoptosis ${ }^{25}$ and is important for ischemic preconditioning, ${ }^{26}$ chronic elevation of IL-6 is associated with $\mathrm{LV}$ dilation and dysfunction. ${ }^{17,27,28} \mathrm{LV}$ samples from the infarct border zone show elevated levels of IL- $6{ }^{29}$ which correlates with LV size after myocardial infarction. ${ }^{30}$ Interestingly, IL- 6 deletion did not prevent or worsen LV dysfunction and adverse remodeling in myocardial infarcted hearts. ${ }^{31}$ These results, together with our findings in this study, demonstrate that IL-6 expression is not required for LV hypertrophy, dilation and dysfunction in pathologically stressed hearts, although chronic elevation of IL-6 is sufficient to induce LV remodeling and dysfunction.

In conclusion, IL-6 deletion does not block LV remodeling and dysfunction in pressure-overloaded hearts. These data suggest that limiting availability of IL-6 alone (such as with immunodepletion with IL-6 antibody) is not sufficient to attenuate LV remodeling and dysfunction in failing hearts.

Supplementary Information accompanies the paper on the Laboratory Investigation website (http://www.laboratoryinvestigation.org)

\section{ACKNOWLEDGEMENT}

This work was supported by Grants from the American Heart Association Western State Affiliates and the National Institutes of Health.

\section{DISCLOSURE/CONFLICT OF INTEREST}

The authors declare no conflict of interest.

1. Lloyd-Jones D, Adams RJ, Brown TM, et al. Heart disease and stroke statistics - 2010 update: a report from the American Heart Association. Circulation 2010;121:e46-e215.

2. Mann D. Pathophysiology of heart failure. In: Libby $P$, Bonow $R$, Mann D, Zipes D (eds). Braunwald's Heart Disease. A Textbook of Cardiovascular Medicine. Saunders: Philadelphia, 2008, pp 541-560.

3. Bozkurt B, Mann DL, Deswal A. Biomarkers of inflammation in heart failure. Heart Fail Rev 2010;15:331-341.
4. Birner CM, Ulucan C, Fredersdorf S, et al. Head-to-head comparison of BNP and IL-6 as markers of clinical and experimental heart failure: superiority of BNP. Cytokine 2007;40:89-97.

5. Tsutamoto T, Asai S, Tanaka T, et al. Plasma level of cardiotrophin-1 as a prognostic predictor in patients with chronic heart failure. Eur J Heart Fail 2007;9:1032-1037.

6. Hirota $\mathrm{H}$, Izumi $\mathrm{M}$, Hamaguchi $\mathrm{T}$, et al. Circulating interleukin-6 family cytokines and their receptors in patients with congestive heart failure. Heart Vessels 2004;19:237-241.

7. Zolk $\mathrm{O}, \mathrm{Ng} \mathrm{LL}, \mathrm{O}$ 'Brien RJ, et al. Augmented expression of cardiotrophin-1 in failing human hearts is accompanied by diminished glycoprotein 130 receptor protein abundance. Circulation 2002;106: 1442-1446.

8. Kalogeropoulos A, Georgiopoulou V, Psaty BM, et al. Inflammatory markers and incident heart failure risk in older adults: the Health $A B C$ (Health, Aging, and Body Composition) study. J Am Coll Cardiol 2010; 55:2129-2137.

9. Matsubara J, Sugiyama S, Nozaki T, et al. Pentraxin 3 is a new inflammatory marker correlated with left ventricular diastolic dysfunction and heart failure with normal ejection fraction. J Am Coll Cardiol 2011; 57:861-869.

10. Kubota T, Miyagishima M, Alvarez RJ, et al. Expression of proinflammatory cytokines in the failing human heart: comparison of recentonset and end-stage congestive heart failure. J Heart Lung Transplant 2000;19:819-824.

11. Cesari $M$, Penninx BW, Newman $A B$, et al. Inflammatory markers and onset of cardiovascular events: results from the Health $A B C$ study. Circulation 2003;108:2317-2322.

12. Fisman EZ, Benderly $M$, Esper RJ, et al. Interleukin- 6 and the risk of future cardiovascular events in patients with angina pectoris and/or healed myocardial infarction. Am J Cardiol 2006;98:14-18.

13. Ohtsuka T, Hamada M, Inoue $K$, et al. Relation of circulating interleukin6 to left ventricular remodeling in patients with reperfused anterior myocardial infarction. Clin Cardiol 2004;27:417-420.

14. Yan AT, Yan RT, Cushman $M$, et al. Relationship of interleukin-6 with regional and global left-ventricular function in asymptomatic individuals without clinical cardiovascular disease: insights from the Multi-Ethnic Study of Atherosclerosis. Eur Heart J 2010;31:875-882.

15. Finkel MS, Oddis CV, Jacob TD, et al. Negative inotropic effects of cytokines on the heart mediated by nitric oxide. Science 1992;257: 387-389.

16. Villegas S, Villarreal FJ, Dillmann WH. Leukemia inhibitory factor and interleukin-6 downregulate sarcoplasmic reticulum Ca2+ ATPase (SERCA2) in cardiac myocytes. Basic Res Cardiol 2000;95:47-54.

17. Janssen SP, Gayan-Ramirez G, Van den Bergh A, et al. Interleukin-6 causes myocardial failure and skeletal muscle atrophy in rats. Circulation 2005;111:996-1005.

18. Melendez GC, McLarty JL, Levick SP, et al. Interleukin 6 mediates myocardial fibrosis, concentric hypertrophy, and diastolic dysfunction in rats. Hypertension 2010;56:225-231.

19. Coles B, Fielding CA, Rose-John S, et al. Classic interleukin- 6 receptor signaling and interleukin-6 trans-signaling differentially control angiotensin II-dependent hypertension, cardiac signal transducer and activator of transcription-3 activation, and vascular hypertrophy in vivo. Am J Pathol 2007;171:315-325.

20. Tang T, Lai NC, Hammond HK, et al. Adenylyl cyclase 6 deletion reduces LV hypertrophy, dilation, dysfunciton and fibrosis in pressureoverloaded female mice. J Am Coll Cardiol 2010;55:1476-1486.

21. Takahashi T, Tang T, Lai NC, et al. Increased cardiac adenylyl cyclase expression is associated with increased survival after myocardial infarction. Circulation 2006;114:388-396.

22. Tamariz L, Hare JM. Inflammatory cytokines in heart failure: roles in aetiology and utility as biomarkers. Eur Heart J 2010;31:768-770.

23. Kimura R, Maeda M, Arita A, et al. Identification of cardiac myocytes as the target of interleukin 11, a cardioprotective cytokine. Cytokine 2007;38:107-115.

24. Obana $M$, Maeda $M$, Takeda $K$, et al. Therapeutic activation of signal transducer and activator of transcription 3 by interleukin-11 ameliorates cardiac fibrosis after myocardial infarction. Circulation 2010;121:684-691.

25. Matsushita K, Iwanaga S, Oda T, et al. Interleukin-6/soluble interleukin6 receptor complex reduces infarct size via inhibiting myocardial apoptosis. Lab Invest 2005;85:1210-1223. 
26. Dawn B, Xuan YT, Guo Y, et al. IL-6 plays an obligatory role in late preconditioning via JAK-STAT signaling and upregulation of iNOS and COX-2. Cardiovasc Res 2004;64:61-71.

27. Kurdi M, Booz GW. Can the protective actions of JAK-STAT in the heart be exploited therapeutically? Parsing the regulation of interleukin-6-type cytokine signaling. J Cardiovasc Pharmacol 2007; 50:126-141.

28. Lecour S, James RW. When are pro-inflammatory cytokines SAFE in heart failure? Eur Heart J 2011;32:680-685.
29. Gwechenberger M, Mendoza LH, Youker KA, et al. Cardiac myocytes produce interleukin- 6 in culture and in viable border zone of reperfused infarctions. Circulation 1999;99:546-551.

30. Ono K, Matsumori A, Shioi T, et al. Cytokine gene expression after myocardial infarction in rat hearts: possible implication in left ventricular remodeling. Circulation 1998;98:149-156.

31. Fuchs $M$, Hilfiker $A$, Kaminski $K$, et al. Role of interleukin- 6 for LV remodeling and survival after experimental myocardial infarction. FASEB J 2003;17:2118-2120. 